\title{
Ventricular Arrhythmias in Postoperative Tetralogy of Fallot
}

\author{
Jay S. Chandar, MD, MRCP, Grace S. Wolff, MD, Arthur Garson Jr., MD, Timothy J. Bell, MD, \\ Stanley D. Beder, MD, Margreet Bink-Boelkens, MD, Craig J. Byrum, MD, \\ Robert M. Campbell, MD, Barbara J. Deal, MD, Macdonald Dick II, MD, Celia J. Flinn, MD, \\ Winston E. Gaum, MD, Paul C. Gillette, MD, Allan J. Hordof, MD, John D. Kugler, MD, \\ Co-burn J. Porter, MD, and Edward P. Walsh, MD
}

\begin{abstract}
Ventricular arrhythmias in patients after total surgical repair of tetralogy of Fallot have been associated with late sudden death. In this large multicenter retrospective study of 359 patients with postoperative tetralogy of Fallot, spontaneous ventricular premature complexes (VPCs) on 24hour ambulatory electrocardiographic monitoring and laboratory-induced ventricular tachycardia (VT) by electrophysiologic stimulation were analyzed. The mean age at surgical repair was 5 years and the mean follow-up duration after repair was 7 years. Spontaneous VPCs on ambulatory monitoring were found in $\mathbf{4 8 \%}$ and induced VT on electrophysiologic stimulation was found in $17 \%$ of patients. Both spontaneous VPCs and Induced VT were significantly related to delayed age at repair, longer follow-up interval, symptoms of syncope or presyncope and right ventricular systolic hypertension $(>60 \mathrm{~mm} \mathrm{Hg})(p<0.05)$, but not to right ventricular diastolic pressure $>8 \mathrm{~mm} \mathrm{Hg}$. The VPCs on ambulatory monitoring were more complex with increasing age at repair and follow-up duration. Induction of VT on electrophysiologic stimulation correlated with spontaneous VPCs including VT on 24-hour ambulatory electrocardiographic monitoring. The electrophysiologic stimulation protocol varied and the induction of VT increased with a more aggressive stimulation protocol. While induced sustained monomorphic VT was related to all forms of spontaneous VPCs, induced nonsustained polymorphic VT was related to more complex forms of VPCs on ambulatory monitoring. VT was not induced in asymptomatic patients who had normal 24-hour ambulatory electrocardiographic monitoring and normal right ventricular systolic pressure. Late sudden death occurred in 5 patients, most of whom had spontaneous VPCs on ambulatory monitoring and right ventricular diastolic pressure $>8 \mathbf{~ m m ~ H g}$, but none had induced VT with a nonaggressive electrophysiologic protocol.
\end{abstract}

(Am J Cardiol 1990;65:655-661)
$\mathrm{S}$ urgical repair of tetralogy of Fallot results in improved duration and quality of life. The 10-year survival rate is $>90 \% .^{1,2}$ However, sudden death is a well-recognized late event with a reported incidencc as high as $4.6 \% .^{1-4}$ Late sudden death is attributed to various cardiac arrhythmias including progressive conduction disturbance with late complete heart block, $, 3,5,6$ sinus node dysfunction? and ventricular premature complexes (VPCs)..$^{8-15}$ These reports focus on VPCs as the primary etiology of late sudden death. Older age at surgical repair, longer interval after repair and poor right ventricular hemodynamic status are considered additional risk factors for late sudden death. ${ }^{16-18}$ Induction of ventricular tachycardia (VT) by electrophysiologic stimulation has been associated with spontaneous VPCs in children with postoperative tetralogy of Fallot. ${ }^{4,15,16,19}$ This large multicenter retrospective study in patients with postoperative tetralogy of Fallot had 2 purposes: to analyze and relate spontaneous VPCs to laboratory-induced VT on electrophysiologic stimulation, and to investigate the prognostic significance of these factors to sudden death.

\section{METHODS}

Fifteen centers participated in this collaborative study. We included 359 patients who had surgical repair before 20 years of age. All patients had an electrophysiologic study that included programmed stimulation at the right ventricular outflow or apex. Stimulation protocol varied among the centers and included single, double or triple extrastimuli and burst pacing.

Data collection: The following were recorded in the 359 patients and analyzed: age at surgical repair, age at

From the University of Miami, Miami, Florida; Baylor College of Medicine, Houston, Texas; Case Western Reserve University, Cleveland, Ohio; University of Groningen, Groningen, The Netherlands; SUNY Health Science Center, Syracuse, New York; Vanderbilt University, Nashville, Tennessee; University of Illinois College of Medicine, Chicago, Illinois; University of Michigan, Ann Arbor, Michigan; Arizona Pediatric Heart Specialists, Phoenix, Arizona; Children's Hospital, Cincinnati, Ohio; Medical University of South Carolina, Charleston, South Carolina; Columbia University, New York, New York; University of Nebraska, Omaha, Nebraska; Mayo Clinic, Rochester, Minnesota; and Harvard Medical School, Boston, Massachusetts. This study was supported in part by an Investigatorship Award, American Heart Association, Broward County Chapter, Inc., Florida. Manuscript received June 26, 1989; revised manuscript received and accepted October 30,1989 .

Address for reprints: Jay S. Chandar, MD, MRCP, University of Miami School of Medicine, Division of Pediatric Cardiology, PO Box 016960 (R-76), Miami, Florida 33101. 


\begin{tabular}{|c|c|c|c|c|c|}
\hline Category & & $\begin{array}{l}\text { Pts } \\
\text { (n) }\end{array}$ & Subgroups & No. & $\%$ \\
\hline \multirow[t]{4}{*}{ Symptoms } & Syncope & 34 & Symptomatic & 53 & 15 \\
\hline & Presyncope & 19 & & & \\
\hline & None & 270 & Asymptomatic & 270 & 75 \\
\hline & Nonspecific & 36 & & & \\
\hline \multirow{5}{*}{$\begin{array}{l}\text { RV hemo- } \\
\text { dynamic } \\
\text { status } \\
\text { (mm Hg) }\end{array}$} & $S P>60 ; D P>8$ & 47 & $\mathrm{SP}>60$ & 76 & 21 \\
\hline & $\mathrm{SP}>60 ; \mathrm{DP}<8$ & 29 & & & \\
\hline & $\mathrm{SP}<60 ; \mathrm{DP}>8$ & 111 & $D P>8$ & 158 & 44 \\
\hline & $\mathrm{SP}<60 ; \mathrm{DP}<8$ & 170 & & & \\
\hline & No study & 2 & Normal & 170 & 47 \\
\hline \multirow{4}{*}{$\begin{array}{l}\text { 24-hour } \\
\text { ambulatory } \\
\text { monitoring }\end{array}$} & VT & 32 & Complex & 77 & 21 \\
\hline & $\mathrm{CP} / \mathrm{MVPC}$ & 45 & & & \\
\hline & UVPC $>10 / \mathrm{hr}$ & 32 & Simple & 97 & 27 \\
\hline & $\begin{array}{l}\text { Normal } \\
\text { No study }\end{array}$ & $\begin{array}{r}129 \\
56\end{array}$ & Normal & 129 & 36 \\
\hline \multirow{4}{*}{$\begin{array}{l}\text { EPS-induced } \\
V T\end{array}$} & All VT & $60^{*}$ & & & 17 \\
\hline & & & SusVT & 33 & 9 \\
\hline & & & NsusMVT & 24 & 7 \\
\hline & & & NsusPVT & 8 & 2 \\
\hline \multicolumn{6}{|c|}{ 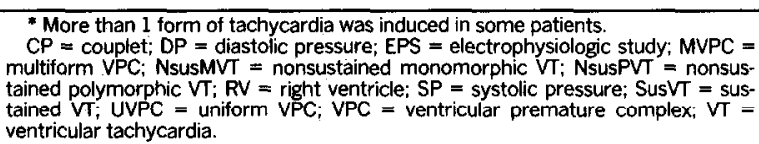 } \\
\hline
\end{tabular}

electrophysiologic study, follow-up interval (time from surgical repair to electrophysiologic study), symptoms of syncope or presyncope, right ventricular systolic and diastolic pressures on hemodynamic study, spontaneous VPCs on 24-hour ambulatory electrocardiographic monitoring and induced VT on electrophysiologic stimulation.

Patient population: The age at surgical repair ranged from 1 to 19 years (mean $4.7 \pm 3.3$ ), the age at electrophysiologic study ranged from 2 to 35 years (mean $12 \pm 7$ ) and the follow-up interval from 0.1 to 28 years (mean $6.9 \pm 5.3$ ). Symptoms of syncope or presyncope were present in $15 \%$, right ventricular systolic hypertension in $21 \%$, diastolic hypertension in $44 \%$, VPCs on 24-hour ambulatory electrocardiographic monitoring in $48 \%$ and induced VT on electrophysiologic stimulation in $17 \%$ of patients (Table I).

Definitions: On 24-hour ambulatory electrocardiographic monitoring, the complex group included multiform VPCs, couplets or VT, the simple group refers to uniform VPCs and the normal group refers to the absence of VPC. VT on ambulatory monitoring or induced VT on electrophysiologic stimulation was defined as $\geq 3$ successive VPCs. Induced sustained VT was defined as VT persisting for $>30$ seconds or requiring termination by electrical stimulation. Nonsustained VT was defined as VT of $>3$ VPCs and terminating spontaneously within 30 seconds. Right ventricular systolic hypertension was defined as pressure $>60 \mathrm{~mm} \mathrm{Hg}$ and diastolic hypertension as $>8 \mathrm{~mm} \mathrm{Hg}$.

Statistical analysis: The data were analyzed by the IBM-compatible Statpac statistical program. Relations between numerical variables were analyzed by the Student $t$ test. Comparisons between variables distributed into categories were made by chi-square analysis. Statistical significance was inferred if $\mathrm{p}$ was $<0.05$. VPCs on monitoring and VT induced on electrophysiologic stimulation were also related to other categories by stepwise logistic regression analysis using the BMD/PLR computer program.

\section{RESULTS}

Symptoms: The patients with symptoms of syncope or presyncope $(15 \%)$ were grouped together and compared to those with no symptoms (75\%) (Table I). Patients with nonspecific symptoms (i.e., chest pain, dizziness etc.) were not included in the analysis. The mean age at repair was $6.4 \pm 4.5$ years in the symptomatic group compared to $4.1 \pm 2.7$ years in the asymptomatic group ( $p<0: 01)$. Similarly, mean follow-up interval (10 \pm 5.9 vs $6 \pm 4.7$ years, $p<0.01$ ) was higher in the symptomatic group. Symptoms of syncope or presyncope did not relate to right ventricular hemodynamic status.

Right ventricular hemodynamic status: The patients with systolic $(21 \%)$ or diastolic hypertension $(44 \%)$ were compared to those with normal pressures (47\%) (Table

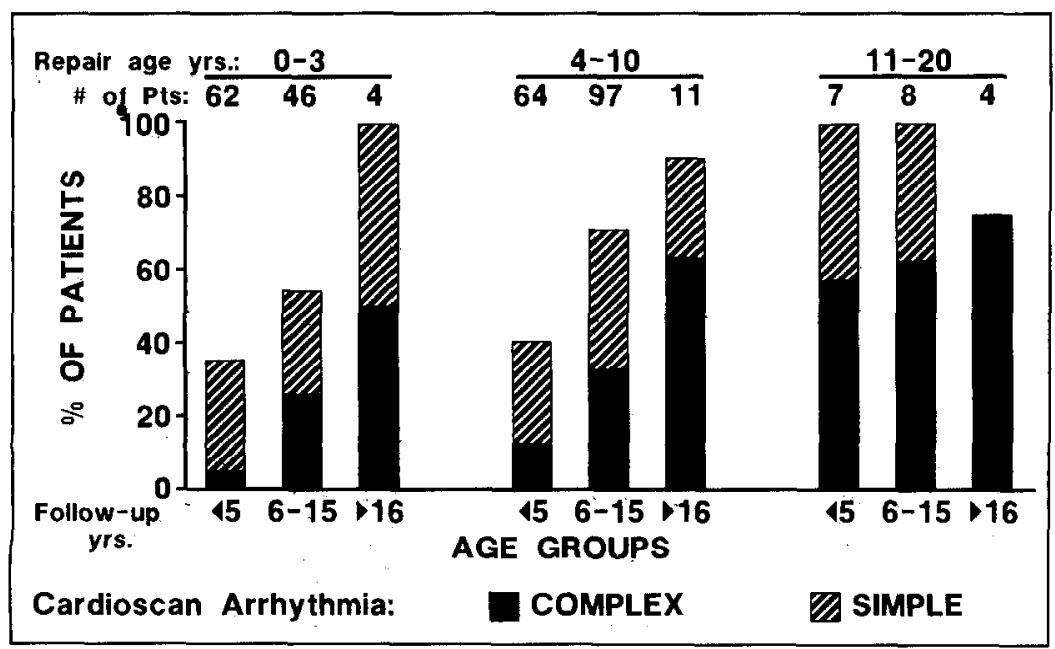

FIGURE 1. Age at repair is divided into 3 age groups, and in each of these groups, follow-up interval is divided into 3 groups. Cardioscan $=24$-hour ambulatory electrocardiographic monitoring. 


\begin{tabular}{|c|c|c|c|c|c|c|c|c|}
\hline \multirow[b]{2}{*}{ Category } & \multirow{2}{*}{$\begin{array}{l}\text { All VPC } \\
(n=174) \\
\text { No. }(\%)\end{array}$} & \multirow{2}{*}{$\begin{array}{l}\text { Complex } \\
(n=77) \\
\text { No. }(\%)\end{array}$} & \multirow{2}{*}{$\begin{array}{l}\text { Simple } \\
(n=97) \\
\text { No. }(\%)\end{array}$} & \multirow{2}{*}{$\begin{array}{l}\text { Normal } \\
(n=129) \\
\text { No. }(\%)\end{array}$} & \multicolumn{4}{|l|}{$\mathrm{p}$ Value } \\
\hline & & & & & $\begin{array}{l}\text { All VPC/ } \\
\text { Normal }\end{array}$ & $\begin{array}{l}\text { Complex/ } \\
\text { Simple }\end{array}$ & $\begin{array}{l}\text { Complex/ } \\
\text { Normal }\end{array}$ & $\begin{array}{l}\text { Simple/ } \\
\text { Normal }\end{array}$ \\
\hline Age at repair (yrs) & $5.9 \pm 4.0$ & $6.8 \pm 4.5$ & $5.2 \pm 3.4$ & $3.7 \pm 2.0$ & $\begin{array}{l}<0.001 \\
<0.001\end{array}$ & 0.011 & $<0.001$ & $<0.001$ \\
\hline Age at EPS (yrs) & $14.7 \pm 7.1$ & $17.3 \pm 7.4$ & $12.7 \pm 6.2$ & $9.0 \pm 5.2$ & $<0.001$ & $<0.001$ & $<0.001$ & $<0.001$ \\
\hline Follow-up interval (yrs) & $8.8 \pm 5.3$ & $10.5 \pm 5.7$ & $7.5 \pm 4.6$ & $5.4 \pm 4.6$ & $<0.001^{*}$ & $<0.001$ & $<0.001$ & 0.001 \\
\hline \multicolumn{9}{|l|}{ Symptoms $(n=269)$} \\
\hline Syn/Presyn $(n=50)$ & $37(74)$ & $22(44)$ & $15(30)$ & $13(26)$ & $\begin{array}{r}0.002 \\
>0.05^{*}\end{array}$ & 0.006 & $<0.001$ & 0.179 \\
\hline Normal $(n=219)$ & $110(50)$ & $37(17)$ & $73(33)$ & $109(50)$ & & & & \\
\hline \multicolumn{9}{|c|}{$R \vee$ pressure $(\mathrm{mm} \mathrm{Hg})(n=301)$} \\
\hline$S P>60(n=65)$ & $45(69)$ & $19(29)$ & $26(40)$ & $20(31)$ & $\begin{array}{r}0.037 \\
>0.05^{*}\end{array}$ & 0.723 & 0.051 & 0.079 \\
\hline$D P>8(n=133)$ & $80(60)$ & $37(28)$ & $43(32)$ & $53(40)$ & 0.412 & 0.356 & 0.147 & 0.603 \\
\hline$S P>60 ; D P>8(n=157)$ & $95(61)$ & $45(29)$ & $50(32)$ & $62(39)$ & 0.264 & 0.269 & 0.100 & 0.604 \\
\hline Normal $(n=144)$ & $77(54)$ & $30(21)$ & $47(33)$ & $67(46)$ & & & & \\
\hline
\end{tabular}

I). The mean age at repair was higher $(5.8 \pm 3.1$ years $)$ in patients with systolic hypertension compared to those with normal pressures $(4.3 \pm 3.3$ years $)(p<0.01)$. Diastolic hypertension did not relate to age at repair.

Ventricular premature complexes on 24-hour monitoring: VPCs on 24-hour ambulatory electrocardiographic monitoring were present in $48 \%$; they were complex in $21 \%$ and simple in $27 \%$ of patients (Table I). Age at surgical repair, follow-up interval and symptoms of syncope or presyncope were greater in patients with VPCs compared to those with normal ambulatory monitoring results $(\mathrm{p}<0.01)$ (Table II). Right ventricular systolic hypertension was more frequent in patients with VPCs $(\mathrm{p}<0.05)$, but right ventricular diastolic hypertension was not related. Stepwise logistic regression analysis related delayed age at repair and increased follow-up interval to VPCs on 24-hour ambulatory electrocardiographic monitoring ( $p<0.01$ ), while symptoms of syncope or presyncope and right ventricular systolic hypertension were not related to VPCs on 24-hour ambulatory electrocardiographic monitoring.

Complex VPCs on ambulatory monitoring were more common with later age at repair and increased follow-up interval (Figure 1). The age at surgical repair was delayed, the follow-up interval was longer and the symptoms of syncope or presyncope were greater in patients with complex VPCs on 24-hour ambulatory electrocardiographic monitoring compared to those with simple VPCs or no VPC on monitoring ( $\mathrm{p} \leq 0.01$ ) (Table II). Similarly, the age at surgical repair and followup interval were greater in patients with simple VPCs compared to those with normal 24-hour ambulatory electrocardiographic monitoring ( $\mathrm{p}<0.01$ ). Right ventricular hemodynamic status did not relate to complexity of VPCs on ambulatory monitoring.

Induced ventricular tachycardias at electrophysiologic study: Sustained or nonsustained VT (all VT combined) was induced in 60 patients (17\%) (Table I). Induced VT (all VT combined) (Table III) was significantly related to delayed age at repair, increased follow-up interval (Figure 2), symptoms of syncope or pre- syncope and VPCs on 24-hour ambulatory electrocardiographic monitoring $(p<0.01)$. Right ventricular systolic hypertension ( $\mathrm{p}<0.05$ ), but not diastolic hypertension, was frequently found in patients with induced VT. With stepwise logistic regression analysis, delayed age at repair, symptoms of syncope or presyncope and VPCs on 24-hour ambulatory electrocardiographic monitoring were related to VT induction $(p<0.05)$. Follow-up interval and right ventricular systolic hypertension were not related.

Induced VT (all VT combined) (Table III) did not differ in patients with complex $(34 \%)$ or simple $(22 \%)$ VPCs on 24-hour ambulatory electrocardiographic monitoring $(p=0.07)$, but induction was increased in both complex and simple groups when compared to those with normal recordings $(9 \%)(\mathrm{p}<0.01)$. The frequency of VT induction (all VT combined) in patients with various VPCs on ambulatory monitoring is shown in Figure 3. This frequency was similar in patients with uniform VPCs or couplets and multiform VPCs (19 to $24 \%$ ), and was increased to $47 \%$ in patients with VT on 24-hour ambulatory electrocardiographic monitoring. Significantly, no VT was induced in asymptomatic patients who had normal 24-hour ambulatory electrocardiographic monitoring (no VPC) and normal right ventricular systolic pressure.

Sustained vemtricular tachycardia: Sustained VT was induced in 33 patients (9\%) (Table I). The relation of induced sustained VT to various categories-delayed age at repair, longer follow-up interval, symptoms of syncope or presyncope, right ventricular systolic hypertension and VPCs on ambulatory monitoring-was similar to that for combined VT ( $p<0.05$ ) (Table III). The induction of sustained VT did not differ in patients with complex $(20 \%)$ or simple VPCs $(11 \%)$ on 24 -hour ambulatory electrocardiographic monitoring $(\mathrm{p}=0.1)$, but was higher in both complex and simple groups compared to patients with normal recordings (4\%) (p $<0.05$ ).

Nonsustained ventricular tachycardias: Nonsustained monomorphic VT was induced in 24 (7\%) and 


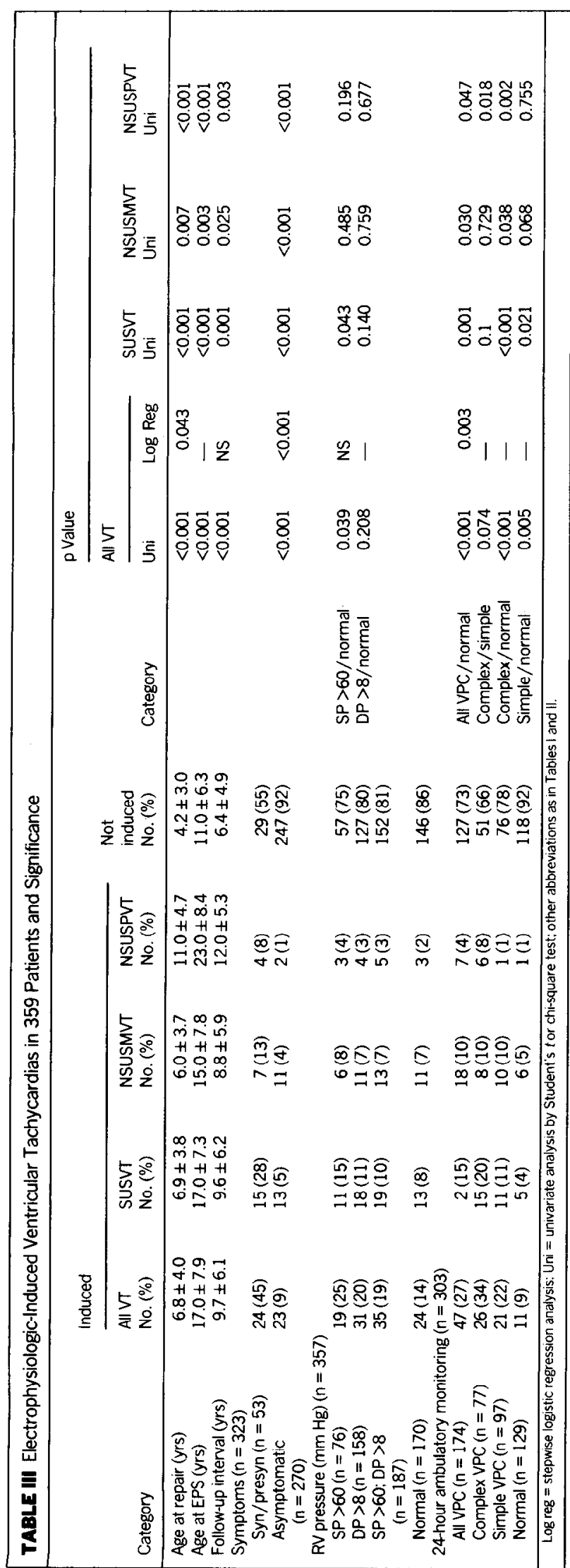

nonsustained polymorphic VT in $8(2 \%)$ patients. The induction of nonsustained VT was related to delayed age at repair, increased follow-up interval, symptoms of syncope and presyncope, and VPCs on ambulatory monitoring ( $p<0.05)$ (Table III). Induction of nonsustained VT did not relate to right ventricular systolic or diastolic hypertension.

The induction of nonsustained monomorphic VT was increased in patients with complex or simple VPCs on 24-hour ambulatory electrocardiographic monitoring (10\%) compared to those with normal monitoring (5\%). The induction of nonsustained polymorphic VT was particularly related to complex VPCs on 24-hour ambulatory electrocardiographic monitoring. Six of 8 patients with induced nonsustained polymorphic VT had complex VPCs on 24-hour ambulatory electrocardiographic monitoring; however, the small number in this group precludes strong conclusions.

Electrophysiologic stimulation protocols: The stimulation protocol varied among different centers. Stimulation was performed in the right ventricular apex in 358 patients (99\%) and right ventricular outflow in 196 patients $(55 \%)$. Single extrastimulation $\left(S_{1} S_{2}\right)$, in apex or outflow, was performed in 356 (99\%), double extrastimuli $\left(\mathrm{S}_{1} \mathrm{~S}_{2} \mathrm{~S}_{3}\right)$ in $272(76 \%)$, triple extrastimuli $\left(\mathrm{S}_{1} \mathrm{~S}_{2} \mathrm{~S}_{3} \mathrm{~S}_{4}\right)$ in $54(15 \%)$ and burst pacing in $246(69 \%)$ patients. VT was induced in 60 patients $(17 \%)$ : in 48 $(13 \%)$ on apex stimulation and in $15(8 \%)$ on outflow stimulation. $\mathrm{S}_{1} \mathrm{~S}_{2}$ induced VT in $12(3 \%), \mathrm{S}_{1} \mathrm{~S}_{2} \mathrm{~S}_{3}$ in 34 $(13 \%), S_{1} S_{2} S_{3} S_{4}$ in $8(15 \%)$ and burst pacing in 18 (7\%) patients.

The stimulation protocols were comparable in patients with VT or complex VPCs, simple VPCs or no VPCs on 24-hour ambulatory electrocardiographic monitoring (Table IV). VT (all VT combined) was induced in 58 of 303 patients who had 24-hour ambulatory electrocardiographic monitoring. (There were 2 additional patients with induced VT who did not have monitoring.) In patients with VT on 24-hour ambulatory electrocardiographic monitoring, $\mathrm{S}_{1} \mathrm{~S}_{2}$ induced VT in $3 \%, S_{1} S_{2} S_{3}$ in $28 \%, S_{1} S_{2} S_{3} S_{4}$ in $33 \%$ and burst pacing in $30 \%$. Aggressive stimulation also increased the frequency of induction of VT in patients with simple VPCs or no VPC on ambulatory monitoring, but to a lesser degree.

Sudden death: Seven patients died of cardiac causes; 1 patient died of chronic heart failure with no arrhythmia and 1 during a repeat surgical procedure. Five patients $(1.4 \%)$ died suddenly. In these 5 patients, the age at surgical repair ranged from 1 to 7 years, and the age of death ranged from 12 to 22 years. VT in 1 and ventricular fibrillation in another patient were documented at death. Retrospective review of 24-hour ambulatory electrocardiographic monitoring showed VT in 1, couplets in 2 and uniform VPCs in 1 patient. Right ventricular diastolic pressure was $>8 \mathrm{~mm} \mathrm{Hg}$ in 4 and right ventricular systolic pressure was $>60 \mathrm{~mm} \mathrm{Hg}$ in 2 patients. During electrophysiologic study, VT was not induced in any of these patients, but the stimulation protocol included double extrastimuli in only 2 and none had triple extrastimuli. 


\begin{tabular}{|c|c|c|c|c|c|}
\hline \multirow{2}{*}{$\begin{array}{l}\text { Stimulation } \\
\text { Protocol/ } \\
\text { Monitoring }\end{array}$} & \multirow[b]{2}{*}{$\begin{array}{l}\text { Attempted } \\
\text { No. }(\%)\end{array}$} & \multicolumn{4}{|c|}{ Induced VT } \\
\hline & & $\begin{array}{l}\text { All VT } \\
\text { No. }(\%)\end{array}$ & $\begin{array}{l}\text { Sus VT } \\
\text { No. (\%) }\end{array}$ & $\begin{array}{l}\text { NsusMVT } \\
\text { No. }(\%)\end{array}$ & $\begin{array}{l}\text { NsusPVT } \\
\text { No. }(\%)\end{array}$ \\
\hline Complex VPCs $(n=77)$ & & $26(34)$ & $15(20)$ & $8(10)$ & $6(8)$ \\
\hline VT $(n=32)$ & & $15(47)$ & $9(28)$ & $5(16)$ & $3(9)$ \\
\hline Single & $31(97)$ & $1(3)$ & $0(0)$ & $1(3)$ & $1(3)$ \\
\hline Double & $29(91)$ & $8(28)$ & $4(14)$ & $3(10)$ & $1(3)$ \\
\hline Triple & $6(19)$ & $2(33)$ & $2(33)$ & $1(17)$ & $0(0)$ \\
\hline Burst & $20(63)$ & $6(30)$ & $4(20)$ & $1(5)$ & $1(5)$ \\
\hline $\mathrm{CP} / \mathrm{MVPC}(\mathrm{n}=45)$ & & $11(24)$ & $6(13)$ & $3(7)$ & $3(7)$ \\
\hline Single & $44(98)$ & $0(0)$ & $0(0)$ & $O(0)$ & $O(0)$ \\
\hline Double & $36(80)$ & $7(19)$ & $3(8)$ & $1(3)$ & $3(8)$ \\
\hline Triple & $3(7)$ & $2(67)$ & $1(33)$ & $1(33)$ & $0(0)$ \\
\hline Burst & $35(78)$ & $4(11)$ & $3(9)$ & $2(6)$ & $O(0)$ \\
\hline Simple VPCs $(n=97)$ & & $21(22)$ & $11(11)$ & $10(10)$ & $1(1)$ \\
\hline UPVC $>10 / h r(n=32)$ & & $6(19)$ & $4(13)$ & $2(6)$ & $0(0)$ \\
\hline Single & $32(100)$ & $O(0)$ & $O(0)$ & $0(0)$ & $0(0)$ \\
\hline Double & $28(88)$ & $5(18)$ & $3(11)$ & $2(7)$ & $0(0)$ \\
\hline Triple & $4(13)$ & $1(25)$ & $1(25)$ & $O(0)$ & $0(0)$ \\
\hline Burst & $22(69)$ & $1(5)$ & $1(5)$ & $O(0)$ & $0(0)$ \\
\hline UPVC $<10 / \mathrm{hr}(n=65)$ & & $15(23)$ & $7(11)$ & $8(12)$ & $1(2)$ \\
\hline Single & $65(100)$ & $6(9)$ & $2(3)$ & $4(6)$ & $0(0)$ \\
\hline Double & $56(86)$ & $5(9)$ & $2(4)$ & $3(5)$ & $1(2)$ \\
\hline Triple & $12(18)$ & $1(8)$ & $0(0)$ & $1(8)$ & $0(0)$ \\
\hline Burst & $55(85)$ & $7(13)$ & $5(9)$ & $2(4)$ & $0(0)$ \\
\hline Normal monitoring $(n=129)$ & & $11(9)$ & $5(4)$ & $6(5)$ & $1(1)$ \\
\hline Single & $129(100)$ & $4(3)$ & $2(2)$ & $2(2)$ & $0(0)$ \\
\hline Double & $83(64)$ & $8(10)$ & $3(4)$ & $4(5)$ & $1(1)$ \\
\hline Triple & $19(15)$ & $2(11)$ & $1(5)$ & $1(5)$ & $0(0)$ \\
\hline Burst & $80(62)$ & $0(0)$ & $O(0)$ & $O(0)$ & $0(0)$ \\
\hline
\end{tabular}

\section{Discussion}

In this collaborative study of 359 patients with surgically repaired tetralogy of Fallot, we have identified significant relations between VPCs on 24-hour ambulatory electrocardiographic monitoring and delayed age at repair, longer time interval after repair, symptoms of syncope or presyncope and right ventricular systolic hypertension. Induction of VT by electrophysiologic stimulation correlated with spontaneous VPCs on 24-hour ambulatory electrocardiographic monitoring. Sudden death was a rare event. Prognostic indicators for sudden death could not be determined by statistical analysis. Nevertheless, there were important features common to the patients who died, namely, VPCs on ambulatory monitoring and right ventricular diastolic hypertension.

Right ventricular hemodynamic status: Several investigators consider the association of right ventricular systolic or diastolic hypertension and VPCs as a significant risk factor for sudden death. ${ }^{2,10,16,17,20}$ This association, however, was not found in reports by Tamer et $\mathrm{al}^{21}$ and Friedli et al. ${ }^{6}$ In this study, right ventricular systolic hypertension was related to VPCs on 24-hour ambulatory electrocardiographic monitoring and induced sustained VT on electrophysiologic study, but was not significant on stepwise logistic regression analysis. Diastolic hypertension was not significant by statistical analysis, but was frequently found in patients with sudden death.
Ventricular arrhythmias on 24-hour ambulatory electrocardiographic monitoring: Several investigators have associated VPCs during electrocardiographic monitoring with late sudden death in patients after repair of tetralogy of Fallot. ${ }^{8-15}$ VPCs of variable severity were found in 20 to $40 \%$ of patients with postoperative tetralogy of Fallot by 24-hour ambulatory electrocardiographic monitoring. $3,13,14,22$ In our study, VPCs were found in $48 \%$ of patients, including VT in $9 \%$ on $24-$ hour ambulatory electrocardiographic monitoring, but our selection of patients is biased by the requirement of an electrophysiologic study.

Ventricular tachycardias induced at electrophysiologic study: The use of electrophysiologic stimulation to identify patients at increased risk for sudden death due to VPCs has been established in adults. ${ }^{23-27}$ The reported electrophysiologic stimulation studies in patients with postoperative tetralogy of Fallot have involved few patients and the prognostic significance of induced VT has not been established. $4,15,16,19$ Deal et al ${ }^{19}$ found electrophysiologic stimulation useful in reproducing clinical ventricular arrhythmia and for selecting effective antiarrhythmic therapy. In our study, VT was induced by varied stimulation protocols in $45 \%$ of patients with syncope or presyncope, in $27 \%$ of patients with VPCs on 24-hour ambulatory electrocardiographic monitoring and in $47 \%$ of patients with VT on monitoring. Conversely, there was no induction of VT in asymptomatic 
patients with normal 24-hour ambulatory electrocardiographic monitoring and normal right ventricular systolic pressure. This combination may be a favorable marker for the patient at low risk.

Induction of sustained monomorphic VT has been accepted as an independent marker of increased risk of sudden death in adult patients. ${ }^{23}$ The prognostic significance of induced sustained VT in children is not known and has not been answered by this study-even though no sudden deaths occurred in patients with induced sustained VT. The induction of sustained VT by varied stimulation protocols was related to the presence of VPCs on 24-hour ambulatory electrocardiographic monitoring, but did not relate to the complexity of the VPCs.

The significance of induced nonsustained monomorphic VT is not known. In our study, the induction of nonsustained monomorphic VT was related to symptoms of syncope or presyncope and VPCs on 24-hour ambulatory electrocardiographic monitoring. It did not differ in patients with uniform VPCs and no VPC on 24-hour ambulatory electrocardiographic monitoring. Induced nonsustained polymorphic VT is considered a nonspecific response to aggressive stimulation protocol in adults. ${ }^{23,28,29}$ However, polymorphic VT has been induced in survivors of sudden death. ${ }^{30}$ Deal et al ${ }^{16}$ suggested that polymorphic VT may be the harbinger of ventricular fibrillation and sudden death in postoperative congenital heart disease. In our study, induced nonsustained polymorphic VT was related to complex VPCs on 24-hour ambulatory electrocardiographic monitoring, although the number of patients in this group was too small for reliable interpretation. This entity may emerge as a possible risk predictor in patients with postoperative tetralogy of Fallot.

In adults, the use of an aggressive stimulation protocol increases the sensitivity for inducing sustained tachycardia, but decreases the specificity. ${ }^{24,28}$ In our study, induction of VT was increased with an aggressive stimulation protocol. Although the stimulation varied, the protocols were comparable in patients with complex and simple VPCs or no VPC on 24-hour ambulatory electrocardiographic monitoring. Hence, the association of induced VT to spontaneous VPCs on 24-hour ambulatory electrocardiographic monitoring is reasonable.

Sudden death: Although the number of patients with sudden death in this series was too small to identify a prognostic marker, VPCs on 24-hour ambulatory electrocardiographic monitoring and right ventricular diastolic hypertension were present in most. Electrophysiologic stimulation of the right ventricle did not result in induction of VT in any of these patients, but the stimulation protocol was not aggressive.

In conclusion, we found that delayed age at surgical repair of tetralogy of Fallot and longer follow-up after repair were associated with increased spontaneous and induced ventricular arrhythmias in later years. Electrophysiologic induction of VT, both sustained and nonsustained, was related to symptoms of syncope or presyncope and spontaneous VPCs. Prognostic significance of induced VT to sudden death could not be determined due to varied stimulation protocols. Aggressive stimulation
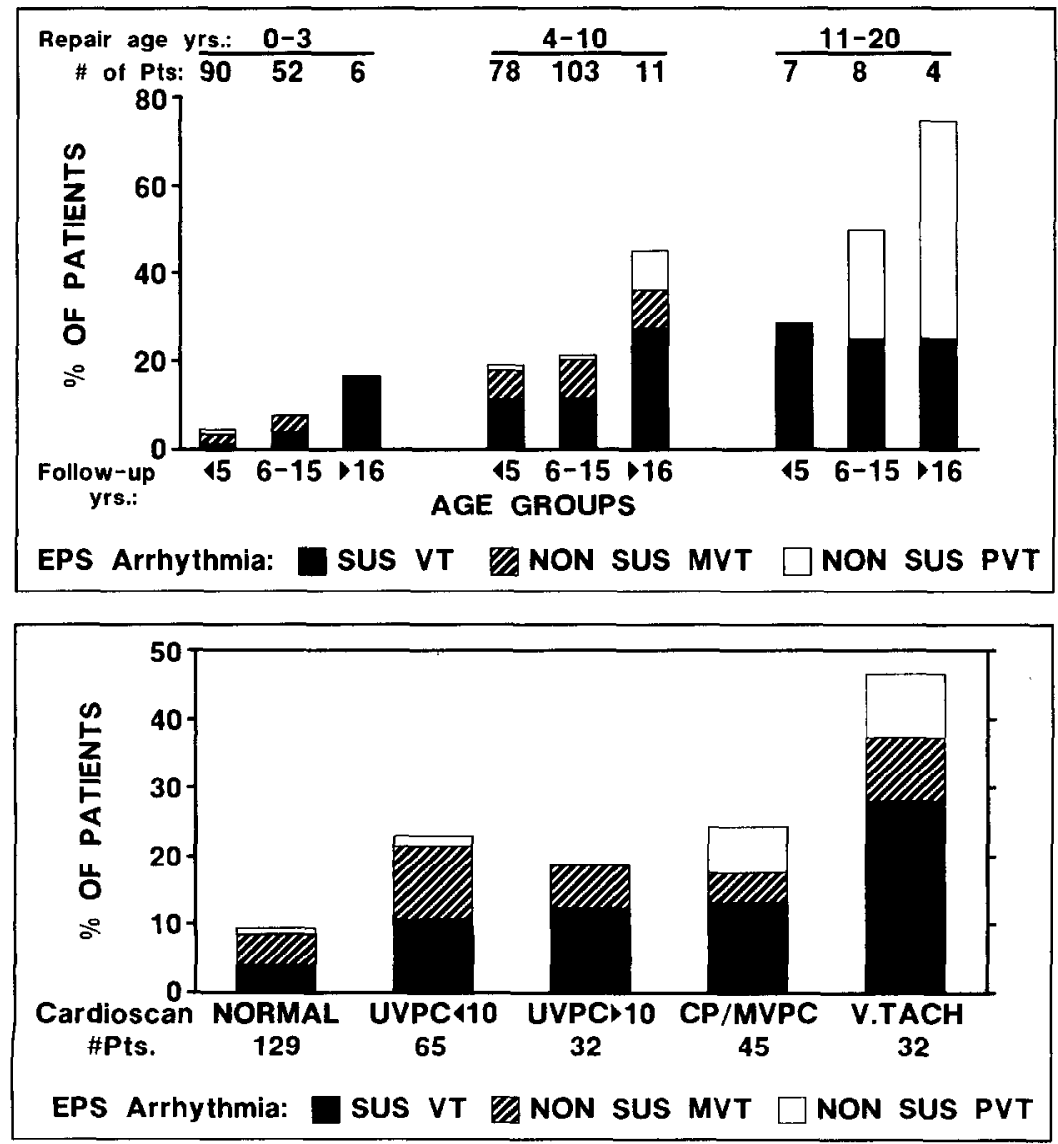

FIGURE 2. Age at repair and follow-up interval are grouped as in Figure 1. EPS = electrophysiologic study; NON SUS MVT = nonsustained monomorphic ventricular tachycardia (VT). NON SUS PVT = nonsustained polymorphic VT; SUS VT = sustained VT.
FIGURE 3. VT induced on electrophysiologic study. CP = couplets, MVPC = multiform ventricular premature complexes, UVPC = uniform ventricular premature complexes, V.TACH = ventricular tachycardia; other abbreviations as in Figure 2. 
protocol increased the sensitivity of VT induction but was performed in only $15 \%$ of patients. The association of spontaneous VPCs and right ventricular dysfunction may be a predictive factor for sudden death.

Prospective studies including aggressive and uniform electrophysiologic stimulation are required to determine the value of electrophysiologic studies in predicting sudden death in postoperative tetralogy of Fallot.

Acknowledgment: We gratefully acknowledge the statistical help and guidance of Robert C. Duncan, $\mathrm{PhD}$, University of Miami. We thank Henry Gelband, MD, University of Miami, for his thoughtful review of this manuscript and Diane McMullen for secretarial assistance.

\section{REFERENCES}

1. Fuster V, McGoon DC, Kennedy MA, Ritter DG, Kirklin JW. Long-term evaluation (12-22 years) of open heart surgery for tetralogy of Fallot. Am J Cardiol 1980:46:635-642.

2. Katz NM, Blackstone EH, Kirklin JW, Pacifico AD, Bargeron LM. Late survival and symptoms after repair of tetralogy of Fallot. Circulation 1982; 65:403-410

3. Deanfield JE, McKenna WJ, Hallidie-Smith KA. Detection of late arrhythmia and conduction disturbance after correction of tetralogy of Fallot. $\mathrm{Br} \mathrm{Hearl} \mathrm{J}$ 1980:44:248-253.

4. Garson A, Porter CJ, Gillette PC, McNarnara DG. Induclion of ventricular tachycardia during electrophysiologic study after repair of tetralogy of Fallot. JACC 1983,6:1493-1502.

5. Wolff GS, Rowland TW, Ellison RC. Surgically induced right bundle-branch block with left anterior hemiblock. An ominous sign in postoperative tetralogy of Fallot. Circulation 1972;46:587-594.

6. Friedli $\mathbf{B}$ Bolens $M$. Taktak $M$. Conduction disturbances after correction of tetralogy of Fallot: are electrophysiologic studies of prognostic value? JACC 1988:11:162-165.

7. Greenwood RG, Rosenthal A, Sloss LJ, LaCorte M, Nadas AS. Sick sinus syndrome after surgery for congenital heart disease. Circulation 1975;52:208213

8. James FW, Kaplan S, Chou T. Unexpected cardiac arrest in patients after surgical correction of tetralogy of Fallot. Circulation 1975;52:691-695.

9. Quattlebaum TG, Varghese J, Neill CA, Donahoo JS. Sudden death among postoperative patients with tetralogy of Fallot. A follow-up study of 243 patients for an averagc of twclve ycars. Circulation 1976;54:289-293

10. Gillette PC, Yeoman MA, Mullins CE, McNamara DG. Sudden death after repair of tetralogy of Fallot. Electrocardiographic and electrophysiologic abnormalities. Circulation 1977;56:566-571.

11. Garson A, Nihill MR, McNamara DG, Cooley DA. Status of the adult and adolescent after repair of tetralogy of Fallot. Circulation 1979:59:1232-I240.

12. Deanfield JE, Ho S, Anderson RH, McKenna WJ, Allwork SP, HallidieSmith KA. Late sudden death after repair of tetralogy of Fallot. A clinicopathologic study. Circulation 1983,67:626-631.

13. Wessel HU, Bastanier CK, Paul MH, Berry TE, Cole RB, Muster AJ Prognostic significance of arrhythmia in tetralogy of Fallot after intracardiac repair. Am J Cardiol 1980:46:843-848.

14. Kavey RW, Blackman MS, Sondheimer HM. Incidence and severity of chronic ventricular arrhythmias after repair of tetralogy of Fallot. Am Heart $J$ 1982:103:342-350.

15. Horowitz LN, Vetter VL, Harken AH, Josephson ME. Electrophysiologic characteristics of sustained ventricular tachycardia occurring after repair of tetralogy of Fallot. Am I Cardiol 1980;46:446-452.

16. Deal BJ, Scagliotti D, Miller SM, Gallastegui JL, Hariman RJ, Levitsky S. Electrophysiologic drug testing in symptomatic ventricular arrhythmias after repair of tetralogy of Fallot. Am J Cardiol 1987:59:1380-1385.

17. Kobayashi J, Hirose H, Nakano S, Matsuda H, Shirakura R. Kawashima $Y$ Ambulatory electrocardiographic study of the frequency and cause of ventricular arrhythmia after correction of tetralogy of Fallot. Am J Cardiol 1984;54:13101313 .

18. Dunnigan A, Pritzker MR, Benditt DL, Benson DW. Life threatening ventricular tachycardias in late survivors of surgically corrected tetralogy of Fallot. $B$ Heart J 1984:52:198-206

19. Kugler JD, Pinsky WW, Cheatham JP, Hofschire PJ, Mooring PK, Fleming WH. Sustained ventricular tachycardia after repair of Tetralogy of Fallot: new clectrophysiologic findings. An J Cardiol 1983;51:1137-1143.

20. Rosing DR, Boser JS, Kent KM, Maron BJ, Seides SF, Morrow AG, Epstein SE. Long-term hemodynamic and electrocardiographic assessment following operative repair of tetralogy of Fallot. Circulation 1978,58(supp/ 1):209-217.

21. Tamer D, Wolff GS, Ferrer P, Pickoff AS, Casta A, Mehta AV, Garcia O. Gelband $\mathrm{H}$. Hemodynamics and intracardiac conduction after operative repair of tetralogy of Fallot. Am J Cardiol 1983;51:552-556.

22. Deanfield JE, McKenna WJ, Presbitero P, England D, Graham GR, Halliedie-Smith K. Ventricular arrhythmia in unrepaired and repaired tetralogy of Fallot. Relation to age, timing of repair, and hemodynamic status. Br Heart $J$ 1984:52:77-81

23. Wellens HJ, Brugada P, Stevenson WG. Programmed electrical stimulation of the heart in patients with life threatening ventricular arrhythmias: what is the significance of induced arrhythmias and what is the correct stimulation protocol? Circulation 1985;72:1-7.

24. Buxton AE, Waxman HL, Marchlinski FE, Undereker WJ, Waspe LE, Josephson ME. Role of triple extrastimuli during electrophysiologic study of patients with documented sustained tachyarrhythmias. Circulation 1984:69:532 540 .

25. Ruskin JN, DiMarco JP, Garan H. Out of hospital cardiac arrest. Electrophysiologic observations and selection of long term antiarrhythmia therapy. $N$ Engl J Med 1980;303:607-6/3.

26. Vandepol CJ, Farshidi A, Spielman SR, Greenspan AM, Horowitz LN. Josephson ME. Incidence and clinical significance of induced ventricular tachycardia. Am J Cardiol 1980:45.725-731.

27. Brugada $\mathbf{P}$, Abdollah $\mathbf{H}$, Heddle B, Wellens HJ. Results of a ventricula stimulation protocol using a maximum of 4 premature stimuli in patients without documented or suspected ventricular arrhythmias. Am J Cardiol 1983:52:1214 1218

28. Brugada P, Green M, Abdollah H, Wellens HJJ. Significance of ventricula arrhythmias initiated by programmed ventricular stimulation: the importance of the type of ventricular arthythmia induced and the number of premature stimuli required Circulation 1984.69:87..92.

29. Stevenson WG, Brugada P, Waldecher B, Zehender M, Wellens HJ. Can potentially significant polymorphic ventricular arrhythmias initiated by programmed stimulation be distinguished from those that are nonspecific? Am Hear $J 1986 \cdot 111: 1073-1080$

30. Benson DW, Benditt DG, Anderson RW, Dunnigan A, Pritzker MR. Cardiac arrest in young, ostensibly healthy patients: clinical, hemodynamic, and electrophysiologic findings. $A m$ J Cardiol 1983;52:65-69. 